\title{
Application of Virtual Reality Technology in Swimming Teaching
}

\author{
https://doi.org/10.3991/ijet.v11i11.6246 \\ Han Guo \\ Northwestern Polytechnical University, China
}

\begin{abstract}
Swimming is one of the most popular spots among college students, though it is hard to master. With virtual reality (VR) technology, problems of the low-cross mutual inductance and low efficiency can be solved. A physical model and the software framework of the virtual reality system was proposed. The swimming teaching system can solve the problem that perception-action separation, improve the study ecological validity, and hold great promise for the study of perception- action. A comparison analysis between students' correct and wrong swimming movements is carried out, and the analysis results show that the virtual reality system solves the problem of monotonicity in real swimming teaching.
\end{abstract}

Index Terms-distributed data, virtual reality technology, swimming teaching

\section{INTRODUCTION}

In the past few years, a way of exchanging information once again become the focus of attention of the industry, that is, virtual reality(VR)technology, especially Facebook with $\$ 20$ billion acquisition of virtual reality helmet manufacturer oculus, instantly improve the market value of the cake, and detonated the whole industry chain. In contrast, the virtual reality technology is only in the stage of scientific research in Colleges and universities, in the stage of general education with virtual reality technology is also facing enormous challenges, The ideal learning environment is requested to be established for the scenario teaching. It can activate the learner's scenario thought, and let the learner's emotion activity participate the perception activity. So that the learner can gain the knowledge, cultivate the ability and develop the intelligence in scenario thought. But a lot of learning environments can't be established in usual teaching activity because of many factors restricted such as time, space or budget etc. The VR is the most suitable technique to establish the ideal learning environment because of its 31 characteristic (immersion, interaction and imagination). This is the best combinative point between the new technical development and the educational development[1-2].

As a major important sport, swimming is one of the most popular sports with college students, bearing the lowest body injury rate. Helping learners to master and improve swimming skills has been an eternal topic that swimming teaching continuously researches and explores. Applying virtual reality in swimming teaching creates a precedent in physical education, bringing a technological leap for swimming teaching.

Due to the poor interaction of traditional swimming teaching method, the efficiency of the usage of swimming teaching resources is low. In order to solve the problem,
Web 2.0 thinking should be used to reform the traditional swimming teaching method.

Having been developed for more than 20 years, the technique of virtual reality (VR) has become one of the most important and inventive 21 th century technologies, paralleling with internet and multi-media. VR is a type of synthetic integration technique, which integrates computer graphics, computer human interaction, sensor technique, artificial intelligence, robotique, etc. and features with multi-sensing, immersion, interactivity and imaginary, etc. Therefore, we can use the virtual reality technology to solve the problem of low cross mutual inductance and low efficiency in tradition swimming teaching.

\section{OVERVIEW}

With the rapid development of computer technology, the researchers are in order to have a deeper research on the technology of virtual reality. Based on the history of the development of virtual reality technology, the system structure of virtual reality technology and the characteristics of virtual reality technology, Li's [3] paper introduces data and architectural visualization, modeling, designing and planning, training and education et. al. applications. And the paper discusses the virtual reality technology may have to need to solve the development problems according to the analysis of the current situation of the development of virtual reality technology summary, Research proves that virtual reality technology is the development of all aspects of life, life will be impact with it in the future.

With the rapid development of economy and science, physical teaching in China has entered a rapid developing period [4]. Tie innovation of physical education has become an urgent demand for further development of physical teaching in new times. Education innovation starting with physical-sport innovation requires the update of education concept and teaching methods. Meanwhile, it is also required to develop students 5 ability of creative thinking to cultivate com-pound sport talents of high quality [5]. Current modem education is based on information technology as background. The combination of information technology with swimming teaching has become a new trend resulted from the innovation of swimming teaching. Such combination actually makes up the inadequacy of traditional teaching methods and means [6].Virtual reality technology is one of the hottest research field both at home and abroad, it has been widely used in the field of education,including miltary colleges and universities teaching, sports special training, aircraft operations, vehicle driving, machine operations, such as teaching. With the development of social productive forces and science and technology,VR technology is growing demand in all kinds of fields. The research of virtual reality and VR software is of vital importance day 
by day. Virtual Reality is a new research paradigm in psychology research area; it has some characteristics such as multi-perception, immersive and interaction. Virtual reality solves the problem that perception and action separating, leads the perception and action study more natural, improves the study ecological validity, and holds great promise for the study of perception- action. In decades, researches about the relationship between perception and action in baseball, handball and tennis found that, athletes can make exact interceptive actions in virtual environment. Johnson's paper [7] analyzes the common function and features of virtual reality software, and compares the advantages and disadvantages of the VR software in order to provide reference for the man developing virtual reality systems.

Swimming is recognized as one of the most popular programs by the whole world in the 21 st century.It can be divided into two categories: competitive swimming and practical swimming. From the perspectiveof the international athletic sports like Olympic Games and Asian Games, where the number of gold medalsof swimming is simply next to that of the track and field events. Moreover, it is widely known that "trackand field, the swimmer the world"; from the perspective of the national fitness, swimming is one of themain sports programs advocated in China's "national fitness program", aiming to promote the sportscompetition and realize its fitness value. However, the relevant researches show that swimming athletictalent is very scarce in China. The sports department of the university, as the main channel of trainingswimming talents, is not optimistic in the teaching situation. Therefore, it is of much necessity and urgencyto reform the teaching situation of swimming in the higher sports colleges [8-11].

\section{METHOD AND ALGORITHM}

Teaching method of physical education is to point to in the sports teaching process, teachers and students to achieve a common goal of physical education teaching, complete the teaching task, and there are plans to take can produce the floorboard of interaction between teaching and learning and teaching activities, is an important part of the overall teaching. The teaching method of using proper directly determines the students to master the movement quality. There are no proper teaching methods, it is difficult for students to better grasp to technology action learning, teaching goals and teaching task is difficult to achieve. In the numerous swimming classes teaching methods and teaching organizational form, every teaching method has characteristics different from other teaching methods; between them also has a certain similarity and association. To improve the quality of teaching, optimize the teaching process, we must in the process of physical education teaching to the teaching method of creative selection and reasonable use. Therefore, a comprehensive comparison of various teaching methods and teaching, in the different stages of the reasonable collocation is the key to solve the problem. The virtual environment and landscape environment mainly includes two parts model environment and real-time roaming environment. And the model environment includes visual modeling and audio modeling. The real-time roaming and the collision detection are realized in real-time roaming environment, which is shown in Figure 1. It adopted parameter key frame and inverse kinematics to simulate swimmers'

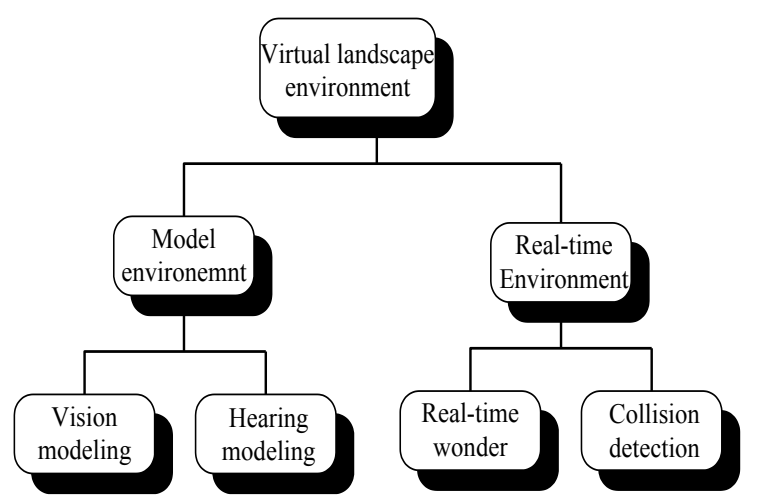

Figure 1. The structure of virtual landscape environment

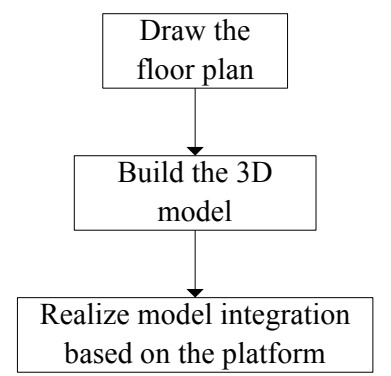

Figure 2. The design process of the virtual reality technology

movements to achieve three-dimensional animation simulation of swimmers. The three-dimensional animation intuitively reproduced standard movements of swimmers in the swimming pool, providing sensory materials for swimming teaching. The work also recorded students' movements through motion detection equipment.

The design process for virtual technology of auxiliary landscape is mainly divided into three steps. As shown in Figure 2, firstly it is the design of the floor landscape plan. The planar layout of the landscape is mainly accomplished by adopting the CAD software and other relevant software design. Then, based on drawing a good plan, through collecting relevant data, the construction of the virtual scene can be achieved. In the virtual scene model, a variety of small models are included, such as building model and road model, model of water level, etc. When building the virtual model, it is needed to collect enough related images, and then the images are made into the picture texture mapping in order to increase the real effect of virtual scene. In order to render the virtual scene, in the process of the scene construction, it is needed to not only meet the effect request, but control the amount of data model. When the landscape model is too big, it is necessary to optimize the virtual model. Finally, the virtual system integration is realized based on the platform. After years of development, the realization of the virtual reality technology is to derive a variety of different methods, such as JAVA3D, CUTE3D, VRML, VRMAP, VRP, etc. The VRP and VRMAP are very good virtual reality software, which are widely used in digital city, remote sensing mapping, geology, petroleum chemical industry, etc. Figure 3 shows the physical model of the system and figure 4 shows the software framework attached with the the physical model of the system. Virtual reality solves the problem that perception and action separating, leads the perception and action study more natural, improves the study ecological validity, and holds great promise for the study of perception- action. 


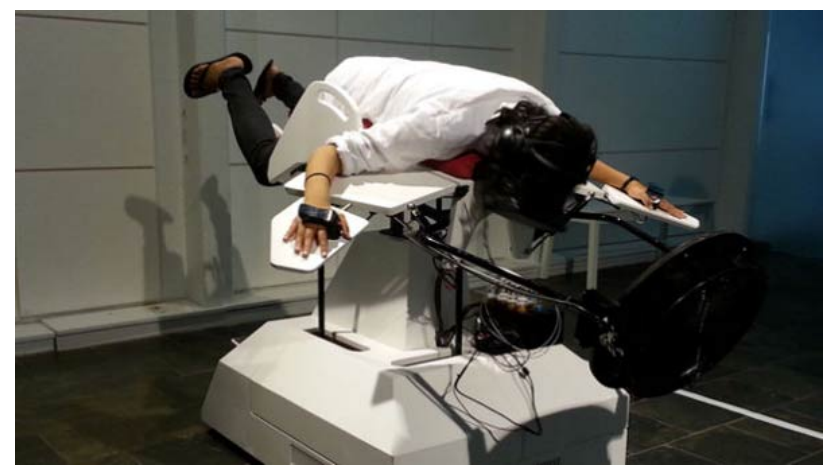

Figure 3. The physical model of the system

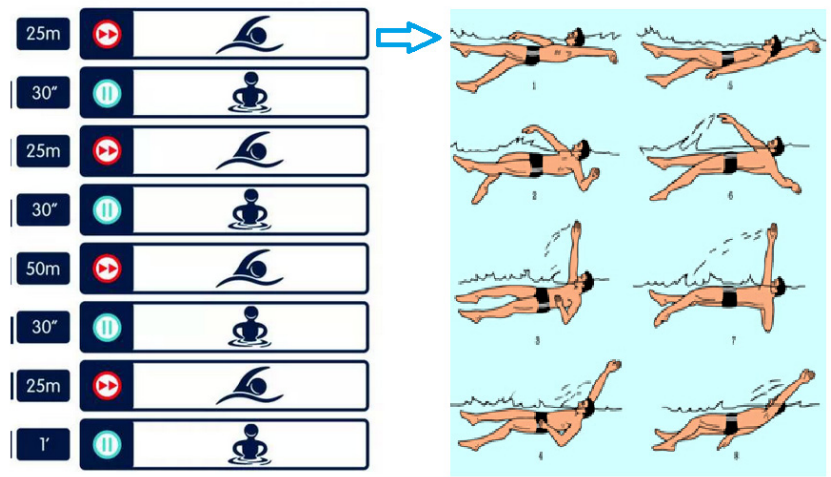

Figure 4. The software framework attached with the physical model of the system

The basic equation of the algorithm is shown in the following equation (1):

In which,

$$
\begin{gathered}
L(\nabla, \omega) f(x, \omega)=0 \\
L(\nabla, \omega)=T(\nabla)+\omega^{2} \rho \mathrm{J}
\end{gathered}
$$

$$
\begin{array}{r}
T(\nabla)=\left\|\begin{array}{cc}
T_{i k}(\nabla) & t_{i}(\nabla) \\
t_{k}^{T}(\nabla) & -\tau(\nabla)
\end{array}\right\|, \quad \mathrm{J}=\left\|\begin{array}{cc}
\delta_{i k} & 0 \\
0 & 0
\end{array}\right\|, \\
f(x, \omega)=\left\|\begin{array}{c}
u_{k}(x, \omega) \\
\varphi(x, \omega)
\end{array}\right\|
\end{array}
$$

Consider delay, the $\mathrm{L}$ can be expressed as:

$$
L^{0}=\left\|\begin{array}{cc}
C_{i j k l}^{0} & e_{k i j}^{0} \\
e_{i k l}^{0 T} & -\eta_{i k}^{0}
\end{array}\right\| \text { (3) }
$$

And local fractional integral of $f(x)$ defined by Eq.4.

$$
\begin{aligned}
& { }_{a} I_{b}^{(\alpha)} f(t)=\frac{1}{\Gamma(1+\alpha)} \int_{a}^{b} f(t)(d t)^{\alpha} \\
& =\frac{1}{\Gamma(1+\alpha)} \lim _{\Delta t \rightarrow 0} \sum_{j=0}^{j=N-1} f\left(t_{\mathrm{j}}\right)\left(\Delta t_{\mathrm{j}}\right)^{\alpha}
\end{aligned}
$$

Its local fractional Hilbert transform, denoted by $f_{x}^{H, \alpha}(x)$ is defined by

$$
\begin{aligned}
& H_{\alpha}\{f(t)\}=\hat{f}_{H}^{\alpha}(x) \\
& =\frac{1}{\Gamma(1+\alpha)} \int_{R} \frac{f(t)}{(t-x)^{\alpha}}(d t)^{\alpha}
\end{aligned}
$$

Where $\mathrm{x}$ is real and the integral is treated as a Canchy principal value, that is,

$$
\begin{aligned}
& \frac{1}{\Gamma(1+\alpha)} \int_{R} \frac{f(t)}{(t-x)^{\alpha}}(d t)^{\alpha} \\
& =\lim _{\varepsilon \rightarrow 0}\left[\frac{1}{\Gamma(1+\alpha)} \int_{-\infty}^{x-\varepsilon} \frac{f(t)}{(t-x)^{\alpha}}(d t)^{\alpha}+(6)\right. \\
& \left.\frac{1}{\Gamma(1+\alpha)} \int_{x+\varepsilon}^{\infty} \frac{f(t)}{(t-x)^{\alpha}}(d t)^{\alpha}\right]
\end{aligned}
$$

To obtain the inverse local fractional Hilbert transform, write again Eq. (7) as

$$
\begin{aligned}
& \hat{f}_{H}^{\alpha}(x)=\frac{1}{\Gamma(1+\alpha)} \int_{-\infty}^{\infty} \frac{f(t)}{(t-x)^{\alpha}}(d t)^{\alpha} \\
& =\frac{1}{\Gamma(1+\alpha)} \int_{-\infty}^{\infty} f(t) g(x-t)(d t)^{\alpha} \\
& =f(x) * g(x),
\end{aligned}
$$

The equation of motion is as follows:

$$
\partial_{j}\left(C_{i j k l} \partial_{k} u_{l}+e_{k i j} \partial_{k} \varphi\right)-\rho \ddot{u}_{i}=0
$$

Under the linear theory, that is:

$$
\partial_{j}\left(e_{i j k l} \partial_{k} u_{l}-\eta_{k i j} \partial_{k} \varphi\right)=0 \text { (9) }
$$

Linear equation can be expressed into the following simplified forms:

$$
\begin{gathered}
L(\nabla, \omega) f(x, \omega)=0, \\
L(\nabla, \omega)=T(\nabla)+\omega^{2} \rho J
\end{gathered}
$$


In which,

Consider delay, the $\mathrm{L}$ can be expressed as:

$$
\begin{gathered}
T(\nabla)=\left\|\begin{array}{cc}
T_{i k}(\nabla) & t_{i}(\nabla) \\
t_{k}^{T}(\nabla) & -\tau(\nabla)
\end{array}\right\|, \mathrm{J}=\left\|\begin{array}{cc}
\delta_{i k} & 0 \\
0 & 0
\end{array}\right\|, \\
f(x, \omega)=\left\|\begin{array}{c}
u_{k}(x, \omega) \\
\varphi(x, \omega)
\end{array}\right\|(12)
\end{gathered}
$$

$$
L^{0}=\left\|\begin{array}{cc}
C_{i j k l}^{0} & e_{k i j}^{0} \\
e_{i k l}^{0 T} & -\eta_{i k}^{0}
\end{array}\right\|
$$

These functions can be expressed in the following form:

$$
\begin{gathered}
C(\mathrm{x})=C^{0}+C^{1}(\mathrm{x}), \quad e(\mathrm{x})=e^{0}+e^{1}(\mathrm{x}), \\
\eta(\mathrm{x})=\eta^{0}+\eta^{1}(\mathrm{x}), \quad \rho(\mathrm{x})=\rho_{0}+\rho_{1}(\mathrm{x})(14)
\end{gathered}
$$

The value with superscript of 1 represents the difference below:

$$
\begin{aligned}
& C^{1}=C-C^{0}, e^{1}=e-e^{0} \\
& \eta^{1}=\eta-\eta^{0}, \rho_{1}=\rho-\rho_{0}
\end{aligned}
$$

The whole function can be simplified into the following integral equation set:

$$
\begin{aligned}
& f(x, \omega)=f^{0}(x, \omega)+\int_{V} S\left(x-x^{\prime}\right)\left(\mathrm{L}^{1} F\left(\mathrm{y}^{\prime}\right)\right. \\
& \left.+\rho_{1} \omega^{2} \mathbf{g}(R) \mathrm{T}_{1} f\left(\mathrm{y}^{\prime}\right)\right] S\left(\mathrm{y}^{\prime}\right) \mathrm{dy}^{\prime}
\end{aligned}
$$

$f(t)$ is defined as:

$$
\left[\frac{\partial}{\partial t}+\varepsilon\right]^{2} f(t)=\delta(t)
$$

The key problem of designing a virtual reality system is how to build a realistic virtual environment, including 3D scene, stereo sound and so on. In the person's feeling, visual data consumes the largest amount of information, accounts for about $70 \%$ of the whole data. The reaction of the visual is also the most agile. If the scene is too simple, the user may feel false. But if the sence is complex, it will increase the difficulty of the interaction, and affect the real-time performance. Virtual landscape visual modeling can be divided into the following three types of modeling terrain model, architecture model, object model.

\section{EXPERIMENT RESULT}

The virtual reality of swimming teaching possesses the characteristics of authenticity, timeliness, extensibility, and richness. Applying virtual reality technology in swimming teaching through computer can accurately reproduce the details of swimming movements in different angles. The software can demonstrate swimming movements in the virtual water environment according to teachers' teaching purpose. Besides, learners can interact with this virtual environment through their natural skills (facial features and limbs). In this way, they can compare their wrong movements with the correct ones in both real and virtual environments, and intuitively make correction at the same time. All-around virtual environment of swimming teaching helps learners to gain the feeling of authenticity. The main function of virtual reality design applied in swimming teaching is to repeat and demonstrate swimming skills and movements in an all-around manner. Following that, a comparison analysis of students 5 correct and wrong movements is carried out. The virtual reality design of swimming teaching includes two plans in practicing: teachers* explanation and demonstration and students, self-study and self-experience. In the former plan, correct swimming skills and movements are displayed in any specific three-dimensional point to strengthen students, understanding of the skills and gestures. In the latter plan, students can intuitively feel the right and wrong movements by themselves. Therefore, they can discover their problems and make corrections in shorter time, improving the learning efficiency.

Average subject characteristics were: age $28.3 \pm 3.9$ years, body mass (with shoes) $75.9 \pm 11.2 \mathrm{~kg}$, and height $175 \pm 8 \mathrm{~cm}$. Subjects walked on a split-belt instrumented treadmill for $30 \mathrm{~s}$ at their preferred walking speed and wearing their own shoes. Preferred walking speed was $0.97 \pm 0.12 \mathrm{~m} / \mathrm{s}$ with a gait cycle of $1.23 \pm 0.09 \mathrm{~s}$. During walking, kinematic marker data were collected at $100 \mathrm{~Hz}$ via a 16-camera passive marker motion capture system with the marker set described in "Supplementary Material". Ground reaction forces were collected at $1,000 \mathrm{~Hz}$ from load cells in the treadmill.

Figure 5 shows errors in joint angles and muscle forces as a function of the allowed computation time in, respectively, the kinematic solver and the static optimization. Figure 6 shows the results are presented for one representative subject. Arrows indicate the settings that are normally used for real-time analysis.

According to the characteristics of swimming teaching, the work put forward theory design for the overall plan of swimming teaching virtual reality. It is actually the collective wisdom from experts and scholars specialized in swimming teaching and computer software design. Moreover, the key technologies, difficulties and detailed implementation procedures were also identified in the work.

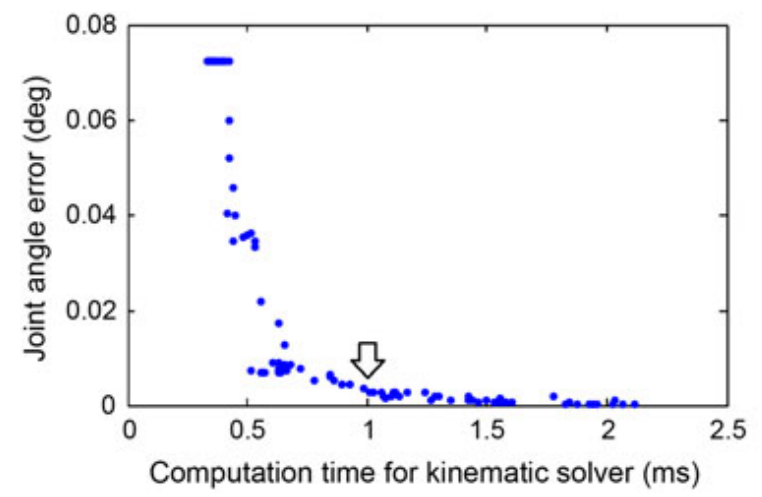

Figure 5. The errors in joint angles and muscle forces as a function of the allowed computation time in, respectively, the kinematic solver and the static optimization

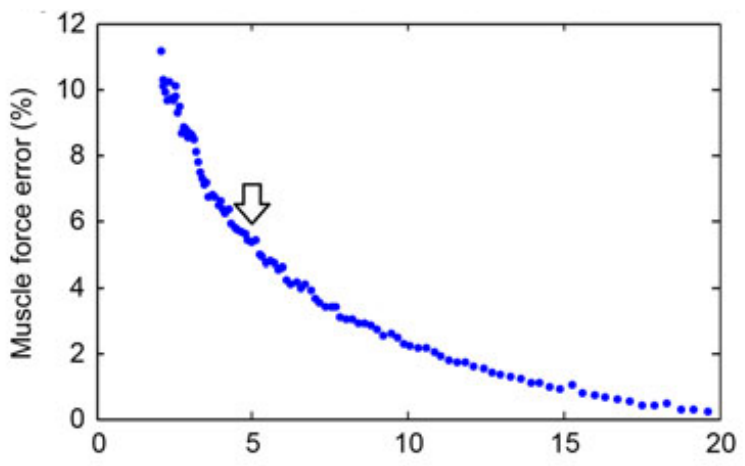

Computation time for static optimization solver (ms)

Figure 6. The results are presented for one representative subject. 


\section{DISCUSSION}

The work mainly studied the application of virtual reality in swimming teaching, explored the virtual reality software for swimming teaching, and preliminarily experimented its teaching effect. Visual environment was combined within computer animation based on detailed researches of virtual swimmer modeling and motion control technology. Then it adopted parameter key frame and inverse kinematics to simulate swimmers movements so as to achieve three-dimensional animation simulation of swimmers. The three-dimensional animation intuitively reproduced the standard movements of swimmers in the swimming pool, thus providing sensory materials for swimming teaching. Then, experimental methods were adopted to conduct teaching experiment based on virtual reality software designed for swimming teaching. In the end, the timeliness of virtual reality in swimming teaching can be verified. At the same time, extensive comments and suggestions can be collected from experts, teachers, and students in the process of experiment. Finally, the work comprehensively analyzed the experimental results, summed up the amendments, and accordingly adjusted those suggestions in the software.

Modeling virtual reality is to simulate the objects and status of real world in digital space. Methods such as actual measurement, generation, and 3D model construction were adopted to build characters and environment for virtual reality of swimming teaching.

Some devices such as data suit and data gloves can be obtained through standard movements' data on land. Accurate swimming technical data obtained through above devices can be used to simulate swimmers. Besides, video analysis method can also be applied to explore swimmers5 movements in water and to obtain relative parameters of excellent swimmers to produce animation.

Capturing movement data of learners Sensors are used to record three-dimensional movements of real swimmers and the computer will drive the virtual swimmer on the computer screen based on the recorded data. In the virtual swimming teaching with the participation of virtual swimmer, computer software will conduct special processing for the recorded movements of real swimmers and the standard movements of virtual swimmers. Thus, the software can achieve a consistence of the viewpoints and angles when learners watch the movements in videos and observe simulated standard movements. Therefore, both the movements in videos and in simulation can be displayed on the same screen, making the comparison of correct and the wrong movements more intuitive and accurate.

For data processing, 100 frames were averaged from a standing trial for initialization of the subject-specific model. The low-pass filter was set to $6 \mathrm{~Hz}$. Computation time limits for the iterative solvers were set to $1 \mathrm{~ms}$ for inverse kinematics, and $5 \mathrm{~ms}$ for static optimization. HBM was executed under Windows 7 on a $2.4 \mathrm{GHz}$ Intel i5 CPU.

The preliminarily designed virtual reality system is applied in actual swimming teaching for implementation. The running conditions of virtual reality can help to record the input and output response of different data and devices, thus gaining experimental data. The work will propose a subsequent improvement plan for the software according to comments and effect from teachers and learners.

\section{CONCLUSION}

In order to solve the problem of monotonicity in teaching, the virtual reality technology is applied in the swimming teaching. The work mainly studied the application of virtual reality technology in swimming teaching and carried out preliminary experiment accordingly, trying to develop virtual reality software for swimming teaching. Virtual environment was combined with computer animation based on detailed research of virtual swimmer modeling and motion control technology. Moreover, the work made use of 3DS MAX software to construct virtual swimming scene: water environment and swimmer. Then it adopted parameter key frame and inverse kinematics to simulate swimmers' movements to achieve threedimensional animation simulation of swimmers.

The three-dimensional animation intuitively reproduced standard movements of swimmers in the swimming pool, providing sensory materials for swimming teaching. The work also recorded students' movements through motion detection equipment. Following that, a comparison analysis of real environment and virtual environment was carried out through data processing. Then, experimental methods were adopted to carry out teaching experiment based on virtual reality software designed for swimming teaching. Meanwhile, its timeliness was also verified and extensive experience comments and suggestions were collected from experts, teachers, and students in the process of experiment. Virtual Reality is a new research paradigm in psychology research area; it has some characteristics such as multi-perception, immersive and interaction. Virtual reality solves the problem that perception and action separating, leads the perception and action study more natural, improves the study ecological validity, and holds great promise for the study of perception- action. In decades, researches about the relationship between perception and action in baseball, handball and tennis found that, athletes can make exact interceptive actions in virtual environment.

Finally, the work comprehensively analyzed the experimental results, summed up the amendments and accordingly adjusted those suggestions in the software. In a word, the work explored and discussed the application of virtual reality in swimming teaching from the aspects of theory and practice. Then, experimental methods were adopted to carry out teaching experiment based on virtual reality software designed for swimming teaching. The experiment result shows the performance of swimming teaching can be improved by using the visual reality technology.

\section{REFERENCES}

[1] H. Jing, "The Study on the Impact of Data Storage from Accounting Information Processing Procedure," International Journal of Database Theory and Application, vol. 8, no.3, pp. 323-332, June 2015. https://doi.org/10.14257/ijdta.2015.8.3.28

[2] Z. Lv, "Wearable smartphone: Wearable hybrid framework for hand and foot gesture interaction on smartphone," In Proceedings of the IEEE International Conference on Computer Vision Workshops, pp. 436-443, 2013. https://doi.org/10.1109/ iccvw.2013.64

[3] L. Li, "Discussion on How to Eliminate the Fear of the Students in Swimming Teaching," Contemporary Sports Technology, 2015.

[4] Z. Chen, "An Analysis on the Domestic Research Papers Concerning Swimming Teaching in Sports Core Periodicals from 19802009," Contemporary Sports Technology, 2014. 
PAPER

ApPlication of ViRTual REALity TeChNOLOGy In SWIMMing TEACHING

[5] H. N. Zhi, and W. Bao, "Preliminary Study on Penetrating Verbal Contract Learning in College Swimming Teaching," Journal of Anhui Normal University, 2014.

[6] Y. I. Qiang, "Guarantee Measures and Security Strategy in Swimming Teaching in the Concept of Survival Education-a case study of universities in Chongqing," Journal of Chengdu Sport University, 2014.

[7] K. Johnson, L. Fingersh, M. Balas, et al., "Methods for increasing region 2 power capture on a variable speed wind turbine," J Solar Energy Eng, pp.1092-1100, 2004. https://doi.org/10.1115/ $\underline{1.1792653}$

[8] K. E. Johnson, L.Y. Pao, M. J. Balas, et al., "Control of variablespeed wind turbines: Standard and adaptive techniques for maximizing energy capture," IEEE Control Syst Mag, pp.70-81, 2006.

[9] Y. Qi, and Y. Shi, "Using a 3D Technology in the Network Distance Teaching of 'Sports Training'," International Journal of
Emerging Technologies in Learning, vol. 11, no.5, pp. 45-50, May 2016. https://doi.org/10.3991/ijet.v11i05.5693

[10] S. Müller, M. Deicke, and R.W. De Doncker, "Doubly fed induction generator system for wind turbines". IEEE Ind Appl Mag, pp:26-33, 2002. https://doi.org/10.1109/2943.999610

[11] Q. Wang, L.C. Chang, "An intelligent maximum power extraction algorithm for inverter based variable speed wind turbine systems". IEEE Trans Power Electron, pp.1242-1249, 2012.

\section{AUTHOR}

Han Guo is with Northwestern Polytechnical University, China (12132130@qq.com).

Submitted 09 September 2016. Published as resubmitted by the author 24 October 2016. 